\title{
Physics and intrinsic properties
}

\author{
Michael Esfeld \\ University of Lausanne, Department of Philosophy \\ Michael-Andreas.Esfeld@unil.ch
}

(for Robert Francescotti (ed.): Intrinsic properties. Berlin: de Gruyter 2013)

\begin{abstract}
The paper sketches out an ontology of physics in terms of matter being primitive stuff distributed in space and all the properties physics is committed to being dispositions that fix the temporal development of the distribution of matter in space. Whereas such properties can be conceived as intrinsic properties of particles in classical mechanics, in quantum physics, there is a holistic property or structure that relates all matter and that fixes its temporal development.
\end{abstract}

\section{Classical physics and intrinsic properties}

At the end of the "Opticks" (1704), Newton writes:

... it seems probable to me, that God in the Beginning form'd Matter in solid, massy, hard, impenetrable, moveable Particles ...; no ordinary Power being able to divide what God himself made one in the first Creation. ... the Changes of corporeal Things are to be placed only in the various Separations and new Associations and Motions of these permanent Particles. (Question 31, p. 400 in the edition Newton 1952)

According to Newton, matter consists in particles that are distributed in a background space, a particle being a material object that is so small that it is localized at a point in space, thus being indivisible. Hence, some points of space are occupied - a particle is localized at them -, whereas other points are empty. The distribution of matter in a background space develops in a background time. That is to say, as time passes, there is change in which points of space are occupied and which are empty. That change is furthermore such that each particle moves on a continuous trajectory. Consequently, each particle has an identity in time by which it distinguishes itself from all the other particles.

If one adopts a sparse view of physical properties, there is no reason to make use of the notion of properties as far as this basic characterization of matter is concerned - the primitive ontology of Newtonian mechanics, to take up an expression introduced by Goldstein (1998) in another context. Matter is primitive stuff, and it is a primitive matter of fact that some points of space are occupied whereas others are not. There is a good reason for conceiving matter in terms of particles, that is, in terms of points of space being occupied or empty. If one considered matter to be a continuous stuff distributed all over space (that is, gunk), then one would have to maintain that there is more stuff at some points of space and less stuff at others in order to be able to accommodate variation. But it could not be a primitive matter of fact that there is more stuff at some points of space and less at others; a property of the stuff would be needed to account for that difference. However, as I shall argue shortly, all the properties that classical mechanics attributes to matter concern its temporal development, not simply the fact that there is matter. The view of matter consisting in particles can easily pay heed to the fact that there is more matter in some regions of space than in others: in some regions of space, more points are occupied than in others. 
Newton's theory seeks to account for the temporal development of the distribution of matter in space. It does so by starting from a certain stipulation: Newton assumes that a certain form of motion of the particles does not call for an explanation, namely inertial motion, that is, constant motion on a straight line (Newton's first law). Only change in the state of motion, that is, the acceleration of the particles (change in their velocity) has to be accounted for. Newton's theory does so by introducing forces (Newton's second law). ${ }^{1}$ It hence is a second order theory, being concerned with the temporal development of the velocity of the particles, by contrast to a first order theory that would be concerned with the temporal development of the position of the particles (velocity being the first temporal derivative of position, acceleration being the second temporal derivative). The forces, in turn, are traced back to properties of the particles. Thus, in virtue of possessing a mass, the particles exert a force upon each other, namely a force of attraction, that is, gravitation. Mass manifests itself in the mutual attraction of the particles (gravitational mass) as well as in their resistance to acceleration (inertial mass).

In brief, a property is attributed to the particles in the form of mass to account for change in their state of motion. I gloss here over the question of whether or not forces should be admitted as properties in addition to properties of the particles such as their mass. ${ }^{2}$ If one seeks for a parsimonious ontology, there is no reason to recognize forces over and above properties of the particles such as their mass, since given the distribution of mass in space at a time, a certain change of the state of motion of the particles - their acceleration as described by the law of gravitation - ensues.

Is mass an intrinsic property of the particles? A world in which only one point of space is occupied at any time - that is, a world with only one particle - is a possible world of Newtonian mechanics, given that Newton admits a background space and a background time. That one particle would forever continue to be in inertial motion (or to be at rest). Consider the widespread view according to which an intrinsic property is a property that an object possesses independently of being alone in a world or being accompanied by other objects. ${ }^{3}$ On this view, mass counts as an intrinsic property.

However, a world in which there is only one particle with mass would be indiscernible from a world in which there is only one massless particle. In other words, taking the particle to be equipped with the property of mass over and above the fact of a point of space being occupied makes no difference as long as one limits oneself to considering possible worlds in which only one point of space is occupied at a time. But the lack of a difference in the case of a one particle world does not decide against mass being an intrinsic property of the particles.

On the way in which I've introduced the ontology of Newtonian mechanics above, the theory starts with the distribution of matter in a background space, that distribution consisting in the fact that some points of space are occupied by primitive stuff, whereas others are not. Properties then are called for only to account for the form of the temporal development of the distribution of the primitive stuff in space, more precisely, to account for changes in the state of motion of the particles. Properties that are needed in order to do that job are dispositions in

1 See Maudlin (2012, chs. 1-2) for an excellent recent examination.

2 See notably Bigelow, Ellis and Pargetter (1988), Wilson (2007) and Massin (2009) on the ontology of Newtonian forces.

3 See Langton and Lewis (1998 and 2001), as well as Hoffmann-Kolss (2010, first part) for a detailed discussion. 
the sense of properties for which it is essential to play a certain role as described by a law. Hence, the answer to the question of whether mass is an intrinsic property in Newtonian mechanics depends on whether or not dispositions are intrinsic properties.

One can with good reason take dispositions to be intrinsic properties. The fact that it is essential for them to perform a certain role as described by a law - make matter accelerate in a certain manner in the case of mass - does not turn them into relations. Any theory of dispositions has to acknowledge the possibility of situations (possible worlds) in which the disposition in question exists, but is not manifest. That is to say, any theory of dispositions has to allow for situations (possible worlds) in which the disposition in question exists, but which are indiscernible from a situation (possible world) in which the disposition in question does not exist - as in the case of a Newtonian world with one particle that has mass and a Newtonian world with one particle that does not have mass. To put it differently, if one could make a case for the following conclusion, that would constitute a sufficient reason not to take mass to be an intrinsic property in Newtonian mechanics: consider a situation - or a sequence of possible worlds - that starts from there being several particles, but which is such that the number of particles is subsequently reduced to only one; one would have to argue for the conclusion that the one particle that is left has lost its mass as a consequence of the annihilation of the other particles that accompany it. However, I don't know an argument that could establish that conclusion.

Instead of regarding mass as a disposition, one may maintain that it is an intrinsic property, but that it exercises the role of changing the state of motion of the particles as described by the law of gravitation only contingently. That is to say, in other possible worlds, mass plays another role. More precisely, considering two different properties (say, mass and charge), it is possible that they swap their roles: in a world $w_{2}$, mass plays the role that charge plays in the actual world $w_{1}$, and charge plays in $w_{2}$ the role that mass exercises in the actual world $w_{1}$. This view implies that the essence of a property - what the property is - is separated from the role that it exercises in a world as described by a law. But what then is the essence of a property? The answer to this question that is dominant in the literature holds that it is a pure quality, known as quiddity (Lewis 2009); but one may also suggest that properties have a primitive numerical identity (Locke 2012).

However, neither of these answers seems convincing: one may contemplate admitting a primitive numerical identity when it comes to primitive stuff filling space, for that stuff is nothing else but what occupies space, as are Newtonian particles as sketched out above. But if one attributes properties to that stuff, it seems odd to admit a primitive numerical identity for these properties as well, for there would then be no reason to take the primitive stuff that occupies space to be equipped with properties at all; one has a reason to recognize properties of the stuff if and only if one wants something that performs a certain job for the temporal development of the stuff. As far as the view of properties being pure qualities is concerned, the problem is that it is not intelligible what could constitute a purely qualitative difference between two properties (say, mass and charge), given that all the accessible difference that justifies recognizing two different properties consists in a difference in the function that these properties exercise for the temporal development of the distribution of matter in space as expressed by a law.

The same objection applies to the mixed view of properties of Martin (1997) and Heil (2003, ch. 11, and 2012, ch. 4) according to which properties are both qualitative and 
dispositional in one. Again, the question is what could constitute a difference in the qualitative aspect of properties such as mass or charge, or what could constitute a reason for admitting a qualitative difference that accompanies the difference in the role that properties such as mass and charge exercise. Again, one needs properties in one's ontology of the natural world only if one wants something that performs a certain function for the behaviour (that is, the temporal development) of the objects that have the properties in question. But then it is sufficient to admit properties that are dispositions, that is, properties whose essence it is to exercise a certain role as described by a law (see notably Bird 2007).

The view of properties being pure qualities is usually put forward in the context of Humeanism, in order to avoid recognizing objective modality. If one considers properties as dispositions, by contrast, one is committed to admitting objective modality, since a disposition plays the same role in any possible world in which it is instantiated - although it is questionable whether this objective modality amounts to metaphysical necessity or whether there is a genuine dispositional modality that is weaker than metaphysical necessity. ${ }^{4}$ However, if one wants to eschew objective modality, one can do so in a much simpler way than by subscribing to a rather baroque metaphysics of pure qualities, namely by recognizing no physical properties at all: the Humean mosaic is the distribution of primitive stuff - such as particles occupying points of space - throughout the whole of space and time. Laws are the axioms of the description of the distribution of that stuff in space and time that achieves the best balance between simplicity and empirical content..$^{5}$ No properties are needed over and above primitive stuff occupying points of space as truth-makers for laws of nature on this view of laws. In any case, the whole distribution of stuff in space-time has to be accepted as primitive on this view of laws, there being nothing that, given an initial distribution of matter in space, determines the temporal development of that distribution.

Let us turn back to the issue of whether properties such as mass in Newtonian mechanics are intrinsic or relations. One can indeed try to make a case for them being relations along the following line: mass is a property of the particles that manifests itself in the form of mutual attraction among the particles. The strength of the force of gravitational attraction between two objects in space depends on their distance (the force diminishes with the square of the distance), but the force is supposed to be transmitted instantaneously through empty space without a medium. That is why Newton's theory is taken to be committed to action at a distance: the presence of a mass in space at a given time $t$ changes the state of motion of all the objects elsewhere in space at that very $t$.

However, one may question whether the notion of an instantaneous action at a distance makes sense: when there is an object with a certain mass somewhere in space at a given time $t$, the manifestation of its mass is present strictly speaking in all the other objects in the universe at that very instant $t$. Consequently, there is no room for an interaction in the sense of the transmission of something. Instead, the correct analysis is to say that the property of mass, including its manifestation, simply is present in all the objects of the universe at any given time $t$. Consider what van Fraassen points out in another context (the context is the discussion of non-local quantum correlations to which I will turn in the next section): 
To speak of instantaneous travel from $X$ to $Y$ is a mixed or incoherent metaphor, for the entity in question is implied to be simultaneously at $X$ and at $Y$ - in which case there is no need for travel, for it is at its destination already. ... one should say instead that the entity has two (or more) coexisting parts, that it is spatially extended. (van Fraassen 1991, p. 351)

These considerations suggest taking mass to be a relation among the objects in space rather than an intrinsic property of each object. That is to say, there is one instantiation of a holistic property of mass distribution at any $t$ that relates all the objects in the universe and that fixes how each of them changes its state of motion at $t$. This relational view of mass can admit a possible world with only one particle as limiting case: there is exactly one instantiation of mass distribution at any $t$ also in that universe, but since there is only one particle that instantiates the mass distribution, there is no change in its state of motion.

However that may be, in any case, Newton's view of particle interaction at a distance was considered to be an anomaly and was replaced by the view of local action among particles transmitted through fields in the $19^{\text {th }}$ and early $20^{\text {th }}$ century. Thus, according to Maxwell's field theory of electromagnetism, in virtue of instantiating the property of charge, each particle builds up a field, the field propagates with a finite velocity (the velocity of light in vacuum is the upper limit), and it is through the field that the charge of a given particle manifests itself in the change of the state of motion of other particles. As regards mass, Einstein achieves a local field theory of gravitation with the theory of general relativity, by identifying the field through which gravitation propagates with the metric field of space-time. In local field theories, the argument rehearsed above for considering the properties attributed to the particles in order to explain the temporal development of their state of motion - such as mass and charge - as intrinsic properties holds firm. In other words, these theories abandon the Newtonian anomaly of instantaneous action at a distance not in favour of developing the idea of one holistic property being instantiated by all the particles in the universe at any time that determines the temporal development of the state of motion of each particle, but in favour of the idea that each particle instantiates an intrinsic property that manifests itself in the change of the state of motion of other particles through the local propagation of a field.

\section{Quantum physics and intrinsic properties}

Let us now turn to quantum mechanics. Any discussion of the issue of what quantum mechanics tells us about the world faces the problem that on the one hand we have a precise formalism for the calculation of probabilities for measurement outcomes at our disposal, but that on the other hand this formalism does not wear an ontology on its sleeves - it is not possible to read any ontological consequences directly off from the formalism. The following, easily accessible thought experiment suggested by Einstein at the Solvay conference in Brussels in 1927 illustrates this situation (the following presentation is based on the reconstruction of Einstein's argument in Norsen 2005): consider a box prepared in Brussels with exactly one particle inside the box. The box is split in two halves in Brussels, one half is sent to New York, the other half is sent to Tokyo. Suppose that Alice in New York opens the box she receives and finds it to be empty. It then is a fact that there is a particle in the box that Bob receives in Tokyo.

The quantum formalism represents the particle in the box by means of a wave-function. When the box is split and the two halves are sent to New York and to Tokyo, the wavefunction represents the particle in terms of a superposition of its being in the box that travels 
to New York and its being in the box that travels to Tokyo. The operational meaning of this representation is that there is a 50\% chance of finding the particle in the box that travels to New York and a 50\% chance of finding the particle in the box that travels to Tokyo. When Alice in New York opens the box she receives and finds it to be empty, the representation by means of the wave-function changes such that the wave-function represents the particle to be in the box that travels to Tokyo. That sudden change is known as collapse of the wavefunction.

One may try an ontological reading of the wave-function in the sense that it provides a literal and thus complete representation of what happens with the particle in this situation. But one then faces the consequence that the collapse of the wave-function means that Alice's action of opening the box she receives in New York instantaneously brings it about that the particle is localized in the box in Tokyo. That is what Einstein considered to be spooky action at a distance. One may therefore turn to an epistemological reading of the wave-function in the sense that it provides all the information about the temporal development of the particle that we can obtain, without being able to represent the actual trajectory that the particle takes. On this reading, the collapse of the wave-function upon Alice's opening of the box she receives in New York simply represents a change in the state of knowledge of the observer once one box has been opened, we know where the particle is, whereas before opening the box, we were ignorant of where it is. This reading implies that the particle always travels on a classical trajectory, being in one box and not being influenced by whatever operation is performed on the other box. This is Einstein's reading of this thought experiment - according to him the only reading that avoids having to acknowledge spooky action at a distance.

However, that reading has been refuted by Bell's theorem in 1964 (reprinted in Bell 2004, ch. 2). In order to understand that refutation, it is not sufficient to consider Einstein's thought experiment of one particle in a box, but one has to turn to the thought experiment that Einstein published together with Podolsky and Rosen in 1935: that thought experiment is about a situation in which two particles are prepared at a source and sent in opposite directions, with the possibility of measuring at least two different parameters on each particle; these parameters are fixed only shortly before the measurement, that is, when the particles are already separated by a distance in space that can be arbitrarily big. Bell's theorem establishes that it is not possible to account for these correlations on the basis of the particles travelling on classical trajectories so that the preparation of the particle pair at the source of the experiment would constitute the cause of the correlations, that is, a cause in the common past of the two measurement events that accounts for the measured correlations. On the contrary, Bell's theorem proves that the fixing of the parameter and the measurement outcome in one wing of the experiment influences the probabilities for the measurement outcome in the other wing of the experiment, even if the events of fixing the parameter and obtaining a measurement outcome between these two wings are separated by a spacelike interval; thus, no signal travelling at most with the speed of light can connect them. ${ }^{6}$

There is no space in this paper to discuss the different proposals for an ontology of quantum mechanics, and there is no need to do so. For the purpose of this paper, it is sufficient to consider the minimal change to the ontology of classical mechanics that is accessible presentation of Bell's theorem and Maudlin (2011) for a detailed examination. 
necessary in order to accommodate the quantum mechanical algorithm for the calculation of probabilities for measurement outcomes. We can do so on the basis of Bell's own way out of the mentioned dilemma into which an epistemological as well as an ontological reading of the wave-function in the quantum mechanical formalism run - that is, a way that pays on the one hand heed to the fact that classical particle trajectories cannot yield the quantum probabilities and that on the other hand avoids the commitment to spooky action at a distance.

One can reconstruct Bell's reasoning in the form of the following four steps:

(1) Any measurement outcome consists in the fact of something having a precise localization in physical space, such as, for instance, a pointer pointing upwards instead of downwards (see e.g. Bell 2004, p. 166). In other words, any measurement outcome consists in a certain distribution of matter in space.

(2) Macroscopic objects - such as the devices that are used as measurement apparatuses - can be localized in physical space if and only if the microscopic objects that compose them are also localized.

(3) If one adopts common sense realism as well as experimental realism, macroscopic objects are localized even if no one observers them. Hence (from (2)), the microscopic objects that compose macroscopic objects are localized independently of whether or not anyone makes a measurement.

(4) Microscopic objects can be localized when they build up macroscopic objects if and only if they are always localized. Otherwise, one would be committed to spooky action at a distance - a measurement operation at a certain location could then have the effect that a microscopic object instantaneously adopts a precise localization arbitrarily far apart in space.

This reasoning shows that there is no need to abandon the basic or primitive ontology of classical mechanics when passing from classical to quantum mechanics. The basic ontology consists in matter distributed in a background space, more precisely in particles existing at points in space. The particles are primitive stuff: that a particle exists at a point in space simply means that a point is occupied. Consequently, the distribution of matter in space is nothing more than the fact that some points of space are occupied, whereas others are empty.

What has to be changed when passing from classical to quantum mechanics is the law for the temporal development of the distribution of matter in space, since classical trajectories of particles cannot yield the quantum mechanical probabilities for measurement outcomes. It is possible to add simply a specific quantum force to the classical forces in order to obtain particle trajectories that yield the quantum mechanical probabilities for measurement outcomes. This is done in that version of the quantum theory that goes back to Bohm (1952) in which a specific quantum force is admitted, known as quantum potential or pilot wave. ${ }^{7}$ However, one can with good reason object that simply adding a quantum force when passing from classical to quantum mechanics is an ad hoc move: that force acts in a non-local manner, thus amounting to action at a distance, without a treatment being possible that matches the manner in which Einstein turned gravitation into a local interaction in the general theory of relativity. Furthermore, that force violates in any case Newton's third law: it acts on the particles without a reaction from the particles on it. 
Let us therefore go one step back and recall the motivation for taking the primitive stuff in space to be endowed with properties at all. We need the commitment to properties if we want something that determines the temporal development of the distribution of the primitive stuff in space. But it is a particular choice made by Newton and further pursued throughout classical physics to take the properties that do so to be such that they give rise to forces - such as mass giving rise to the force of gravitation, or charge giving rise to the force of electricity and magnetism. In other words, it is a particular choice made by Newton and further pursued throughout classical physics to go for a law of motion that is second order, that is, being about acceleration, namely the temporal development of the velocity of the particles. A much simpler choice would be to examine simply the temporal development of the position of the particles, that is, to put forward a law of motion that is first order, being concerned with what determines the velocity of the particles, given their position. In other words, properties in this case are needed as that which fixes the velocity of the particles given their position.

There indeed is a quantum theory available that implements this choice, namely the dominant contemporary version of the theory going back to Bohm (1952), known as Bohmian mechanics ${ }^{8}$. The Bohmian law for the temporal development of the distribution of matter in space is this one:

$$
\frac{d Q}{d t}=v^{\Psi t}(Q)
$$

In this law, the quantum mechanical wave-function $\Psi$ has the job to determine the velocity of the particles at a time $t$, given their position at $t$. If it has to be acknowledged that we are ignorant of the exact initial particle configuration and if one makes a mathematically precise typicality assumption about that initial particle configuration, one can derive the quantum mechanical algorithm for calculating probabilities for measurement outcomes in Bohmian mechanics. ${ }^{9}$ Consequently, Bohmian mechanics grounds textbook quantum mechanics in the sense that it provides an ontology of the distribution of matter in space and a law for the temporal development of that distribution from which the textbook formalism of quantum mechanics can be deduced.

It is possible to regard the quantum mechanical wave-function as referring to a property, namely a dispositional property of the particles that determines their temporal development by fixing their velocity. ${ }^{10}$ However, the wave-function that figures in equation (1) is the universal wave-function; consequently, $Q$ in equation (1) stands for the configuration of all the particles in the universe. That is to say, the property that fixes the velocity of any particle at a time $t$ given its position at $t$ is not an intrinsic property of that particle, but there is only one instantiation of a holistic property of all the particles at $t$, represented by the universal wave-function at $t$, that determines the velocity of each particle at $t$, given the position of all the particles at $t$. That is how Bohmian mechanics accounts for the non-local correlations brought out by the Einstein-Podolsky-Rosen thought experiment and Bell's theorem. In other words, the trajectory of the particles is not fixed by forces acting locally on the particles, but by a holistic property of all the particles taken together.

See Goldstein (2006) for a brief presentation and the papers in Dürr, Goldstein and Zanghì (2013) for a detailed exposition.

9 See Dürr, Goldstein and Zanghì (2013, ch. 2).

10 See Belot (2012, pp. 77-80) for a sketch and Esfeld et al. (2013) for a detailed argumentation. 
Nonetheless, Bohmian mechanics allows for the introduction of what is known as effective wave-functions, that is, wave-functions that apply to particular local configurations of particles while abstracting from the rest of the universe. Bohmian mechanics thereby is in the position to account for both the non-local correlations as brought out by Bell's theorem and for the classical, local character of the environment with which we are familiar. ${ }^{11}$ However, when it comes to ontology by contrast to adopting an attitude that is sufficient for all practical purposes when dealing with the environment with which we are familiar, the availability of effective wave-functions does of course not mean that the temporal development of the particles is determined by intrinsic or local properties instead of one holistic property that takes all the particles in the universe as its relata. Hence, as far as what is specific to quantum mechanics is concerned, there is no room for intrinsic properties instantiated by the objects in space; instead, there is only one holistic property of all these objects taken together that determines their temporal development.

Coming back to the anomaly of instantaneous action at a distance in Newtonian mechanics, whereas classical field theory overcomes this anomaly by conceiving local action induced by intrinsic properties of the particles building up fields that propagate with a finite velocity, quantum theory can be taken to overcome that anomaly by radicalizing the holism that is already available for Newtonian mechanics: as mentioned at the end of the previous section, one can conceive an ontology of Newtonian mechanics in terms of one instantiation of a holistic property of mass distribution at any time $t$ that relates all the objects in the universe and that fixes how each of them changes its state of motion at $t$. By the same token, one can take the universal wave-function in quantum mechanics to refer to one instantiation of a holistic property that relates all the objects in the universe and that fixes the velocity of each of them, given the position of all the particles.

This holism is more radical than the one that one can contemplate with respect to Newtonian mechanics, since the universal wave-function in quantum mechanics does not represent the distribution of a property of objects (as there is in any case a mass distribution in Newtonian mechanics such that each object has mass), but exactly one instantiation of a holistic property of all the particles taken together. That property determines the velocity of each particle, but it is not a property possessed by each particle - due to the non-separability of the wave-function, there is only one wave-function for the whole particle configuration. Nonetheless, quantum mechanics, like Newtonian mechanics, admits a possible world with only one particle as limiting case: that particle has a wave-function, and that wave-function represents the property of that particle which fixes its temporal development. To put it differently, for any possible world, there is exactly one universal wave-function representing the property that fixes the temporal development of the objects in that world, whatever their number may be.

This holism holds whatever view one adopts with respect to the distribution of matter in space in quantum mechanics. One does not have to take that distribution to consist in particles in the sense of primitive stuff occupying points of space; other proposals notably include a continuous distribution of stuff ${ }^{12}$ and sparsely distributed discrete point events, known as 
flashes, making up no continuous trajectories or worldlines. ${ }^{13}$ In any case, whatever the distribution of matter in space may be, its temporal development is fixed by a holistic and dispositional property instantiated by the matter distribution as a whole and represented by the universal wave-function. That is why quantum physics lends support to the view known as ontic structural realism: that holistic property is a structure, because it relates everything that makes up the distribution of matter in space. However, of course, that property or structure is instantiated by something, namely the distribution of matter in space, in whatever entities that distribution may consist. The only reason for admitting that property or structure is that it does the job of determining the temporal development of the distribution of matter in space and thereby also is able to account for measurement outcomes. ${ }^{14}$

Elaborating on the mentioned holism in terms of ontic structural realism can help to bring out the contrast with action at a distance. Bell's theorem rules out Einstein's epistemological view of the quantum mechanical wave-function, but we are thereby not committed to falling back to what Einstein considers as spooky action at a distance. Bohmian mechanics, conceived as a first order theory without a specific quantum force as sketched out above, can illustrate this issue: there is no question of a direct interaction among the particles in Bohmian mechanics - such an interaction would indeed be action at a distance. Instead of interacting directly with each other, the particles are related through the holistic property or structure that determines the velocity of each of them at a time $t$ given the position of all of them at $t$. There is of course indirect interaction among the particles in that a local change in the arrangement of particles (e.g. fixing a parameter in a Bell-type experiment, opening or closing one slit in a double slit experiment, etc.) can influence the velocity of strictly speaking all the other particles, whatever their distance in space is - but it does so through the mentioned structure or holistic property instantiated by all the particles, by contrast to direct interaction among the particles. Operationally speaking, there is no difference between a second order theory that adds a specific, non-local quantum force to the classical forces and a first order theory that takes the temporal development of the distribution of matter in space (such as the velocity of particles) to be determined by a structure or holistic property instantiated by the matter distribution as a whole; both theories agree in their predictions of measurement outcomes. But operational agreement can go with a profound difference in the ontology of these theories.

In any case, the quantum mechanical wave-function cannot be conceived as representing a field in physical space that mediates the interaction among the particles. The quantum mechanical wave-function is defined on configuration space - a high dimensional mathematical space (if there are $N$ particles, the dimension of configuration space is $3 N$ ) without it being possible to take that wave-function to assign field values to points in physical space (three-dimensional space, or four-dimensional space-time). Nonetheless, an analogy with classical fields is possible, if one adopts the attitude of regarding the formalism of classical field theory as expressing how the dispositional properties of the particles such as their charge and mass perform the function of fixing the temporal development of the velocity of the particles, without subscribing to the ontological commitment of fields existing as

13 See Bell (2004, ch. 22) and Tumulka (2006).

14 See Esfeld and Lam (2011) as well as Esfeld (2013) for setting out ontic structural realism in that sense and references to the discussion about ontic structural realism in general. 
concrete objects in physical space over and above the particles. ${ }^{15}$ In that case, the field value assigned to a given space-time point does not represent a physical property existing at that point, but expresses only the information about what would happen to the motion of a particle if a particle were present at that point. The truth-maker for these counterfactual propositions are the dispositional properties of the particles such as their charge and mass. If one takes fields to be nothing more than the mathematical representation of such dispositional properties of the particles, the fact that the quantum mechanical wave-function can only be conceived as a field on configuration space poses no problem.

When one passes from quantum mechanics to quantum field theory and quantum gravity, the mentioned holism is not only confirmed, but moreover strengthened. In what is known as relativistic quantum field theory, despite its being relativistic, Bell's theorem applies: there are correlations between space-like separated events which cannot be accounted for in terms of local common causes (see Bell 2004, ch. 24). The main change with respect to quantum mechanics is the following one: whereas the quantum mechanical algorithm for calculating probabilities for measurement outcomes can be derived from an ontology that is committed to a fixed number of particles whose trajectories are determined by a holistic property instantiated by the configuration of all the particles, it seems that one has to make room for events of particle creation and annihilation in quantum field theory. Nonetheless, whatever one takes the distribution of matter in space to be, that distribution instantiates as a whole a structure or holistic property that fixes its temporal development and that is represented by the universal wave-function. In other words, there is in any case a good reason in quantum field theory to endorse the commitment to a holistic property of the distribution of matter in space that fixes its temporal development, even if that property no longer provides for an intertemporal identity of particles (as it does in Bohmian mechanics). ${ }^{16}$

In quantum gravity, there no longer is a background space and a background time in which matter is inserted, but space and time are themselves quantum objects. Accordingly, there no longer is a universal wave-function that develops itself in a background time, but the universal wave-function is stationary, the Schrödinger equation being replaced with the Wheeler-deWitt equation. Nonetheless, the universal wave-function can still be regarded as referring to a configuration of elementary objects, such as a configuration of elementary parts of space (or a configuration of elementary parts of space-cum-matter), and representing a holistic property of such a configuration that fixes the transition from one such configuration to the next one such that something approximating the classical space-time of general relativity theory is built up. In such a scenario, the holism that is characteristic of quantum physics is strengthened, since whatever relationship holds between the subsequent configurations of elementary objects is given entirely by the mentioned holistic property that is instantiated by any such configuration and that is represented by the universal, stationary wave-function. ${ }^{17}$

In sum, the crucial difference between classical and quantum mechanics is this one: in classical mechanics, there are dispositional properties of the particles that fix their temporal

15 See Lange (2002) for a good introduction to the discussion about whether or not one should subscribe to an ontological commitment to classical fields.

16 For attempts to formulate a Bohmian quantum field theory, see Bell (2004, ch. 19) and Dürr, Goldstein and Zanghì (2013, ch. 10) as well as Struyve (2011) for an overview of the state of the art.

17 See Dürr, Goldstein and Zanghì (2013, ch. 11) for a sketch of a Bohmian ontology of quantum gravity. 
development in the sense of fixing the temporal development of their velocity (acceleration), and these properties can be conceived as intrinsic properties of each particle. As far as what is specific for quantum physics is concerned, there are no intrinsic properties of particles, but only one structure or holistic property that determines the temporal development of the distribution of matter in space (determining in the case of particles the temporal development of their position by fixing the velocity of each particle). Since Bell's theorem can with reason be conceived as putting a constraint on any future physical theory, there seems to be no prospect of going back to intrinsic properties in the ontology of physics.

\section{References}

Bell, John S. (2004): Speakable and unspeakable in quantum mechanics. Cambridge: Cambridge University Press. Second edition.

Belot, Gordon (2012): “Quantum states for primitive ontologists. A case study". European Journal for Philosophy of Science 2, pp. 67-83.

Belousek, Darrin W. (2003): "Formalism, ontology and methodology in Bohmian mechanics". Foundations of Science 8, pp. 109-172.

Bigelow, John, Ellis, Brian and Pargetter, Robert (1988): “Forces”. Philosophy of Science 55, pp. 614-630.

Bird, Alexander (2007): Nature's metaphysics. Laws and properties. Oxford: Oxford University Press.

Bohm, David (1952): “A suggested interpretation of the quantum theory in terms of 'hidden' variables". Physical Review 85, pp. 166-193.

Dürr, Detlef, Goldstein, Sheldon and Zanghì, Nino (2013): Quantum physics without quantum philosophy. Berlin: Springer.

Einstein, Albert, Podolsky, Boris and Rosen, Nathan (1935): “Can quantum-mechanical description of physical reality be considered complete?” Physical Review 47, pp. 777-780.

Esfeld, Michael (2013): "Ontic structural realism and the interpretation of quantum mechanics". European Journal for Philosophy of Science 3, pp. 19-32.

Esfeld, Michael and Lam, Vincent (2011): "Ontic structural realism as a metaphysics of objects". In: A. Bokulich and P. Bokulich (eds.): Scientific structuralism. Dordrecht: Springer. Pp. 143-159.

Esfeld, Michael, Lazarovici, Dustin, Hubert, Mario and Dürr, Detlef (2013): "The ontology of Bohmian mechanics". Forthcoming in British Journal for the Philosophy of Science 64. Paper available at http://philsci-archive.pitt.edu/9381/

Ghirardi, Gian Carlo, Grassi, Renata and Benatti, Fabio (1995): "Describing the macroscopic world: closing the circle within the dynamical reduction program". Foundations of Physics 25, pp. 5-38.

Goldstein, Sheldon (1998): "Quantum theory without observers". Physics Today 51, March pp. 42-46 and April pp. 38-42.

Goldstein, Sheldon (2006): “Bohmian mechanics”. In: E. N. Zalta (ed.): Stanford Encyclopedia of Philosophy. http://plato.stanford.edu/entries/qm-bohm

Goldstein, Sheldon, Norsen, Travis, Tausk, Daniel Victor and Zanghì, Nino (2011): "Bell's theorem". http://www.scholarpedia.org/article/Bell's_theorem

Heil, John (2003): From an ontological point of view. Oxford: Oxford University Press.

Heil, John (2012): The universe as we find it. Oxford: Oxford University Press.

Hoffmann-Kolss, Vera (2010): The metaphysics of extrinsic properties. Frankfurt (Main): Ontos.

Holland, Peter R. (1993): The quantum theory of motion. An account of the de Broglie-Bohm causal interpretation of quantum mechanics. Cambridge: Cambridge University Press.

Lange, Marc (2002): An introduction to the philosophy of physics. Oxford: Blackwell.

Langton, Rae and Lewis, David (1998): "Defining 'intrinsic"”. Philosophy and Phenomenological Research 58, pp. 333-345.

Langton, Rae and Lewis, David (2001): “Marshall and Parsons on intrinsic”. Philosophy and Phenomenological Research 63, pp. 353-355. 
Lewis, David (1973): Counterfactuals. Oxford: Blackwell.

Lewis, David (2009): "Ramseyan humility". In: D. Braddon-Mitchell and R. Nola (eds.): Conceptual analysis and philosophical naturalism. Cambridge (Massachusetts): MIT Press. Pp. 203-222.

Locke, Dustin (2012): “Quidditism without quiddities”. Philosophical Studies 160, pp. 345-363.

Martin, C. B. (1997): “On the need for properties: the road to Pythagoreanism and back”. Synthese 112, pp. 193231.

Massin, Olivier (2009): “The metaphysics of forces”. Dialectica 63, pp. 555-589.

Maudlin, Tim (2011): Quantum non-locality and relativity. Third edition. Chichester: Wiley-Blackwell.

Maudlin, Tim (2012): Philosophy of physics. Volume 1. The arena: space and time. Princeton: Princeton University Press.

Monton, Bradley (2004): "The problem of ontology for spontaneous collapse theories". Studies in History and Philosophy of Modern Physics 35, pp. 407-421.

Mumford, Stephen and Anjum, Rani Lill (2011): Getting causes from powers. Oxford: Oxford University Press.

Newton, Isaac (1952): Opticks or a treatise of the reflections, refractions, inflections and colours of light. Edited by I. B. Cohen. New York: Dover.

Norsen, Travis (2005): “Einstein's boxes”. American Journal of Physics 73, pp. 164-176.

Struyve, Ward (2011): "Pilot-wave approaches to quantum field theory". Journal of Physics: Conference Series 306, p. 012047.

Tumulka, Roderich (2006): “A relativistic version of the Ghirardi-Rimini-Weber model”. Journal of Statistical Physics 125, pp. 825-844.

van Fraassen, Bas C. (1991): Quantum mechanics: an empiricist view. Oxford: Oxford University Press.

Wilson, Jessica (2007): “Newtonian forces”. British Journal for the Philosophy of Science 58, pp. 173-205. 